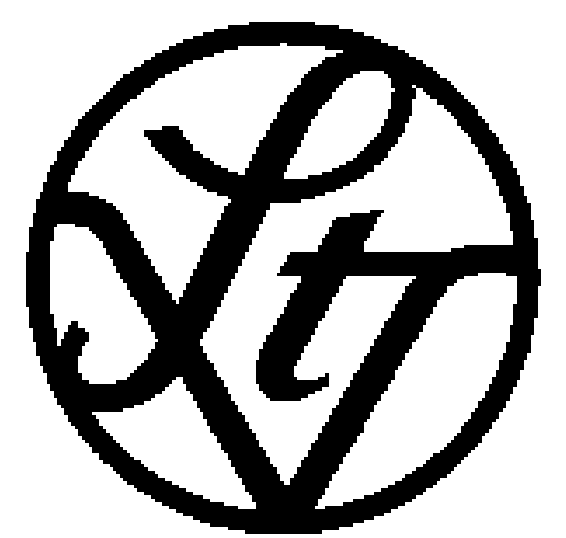

Der metrifche Taktfuß in der modernen Mufik

Author(s): Jlmari Krohn

Source: Archiv für Musikwissenschaft, 4. Jahrg., H. 1. (Feb., 1922), pp. 100-108

Published by: Franz Steiner Verlag

Stable URL: http://www.jstor.org/stable/929843

Accessed: 18/06/2014 07:35

Your use of the JSTOR archive indicates your acceptance of the Terms \& Conditions of Use, available at http://www.jstor.org/page/info/about/policies/terms.jsp

JSTOR is a not-for-profit service that helps scholars, researchers, and students discover, use, and build upon a wide range of content in a trusted digital archive. We use information technology and tools to increase productivity and facilitate new forms of scholarship. For more information about JSTOR, please contact support@jstor.org.

Franz Steiner Verlag is collaborating with JSTOR to digitize, preserve and extend access to Archiv für Musikwissenschaft. 


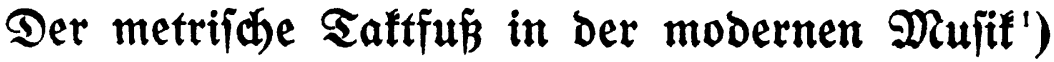

Bon

\author{
Slmari Rrohn, Selfingfors
}

C.8 wirb allgemein anertant, daj in ber antifen Runjt Poefie uno Mujit burd ibren genteinfamell Ngytgmus eng miteinander verbunden waren. Derjelbe gründete fin auf metrifde Bejege, bie fid in ber gefamten Reibe ber

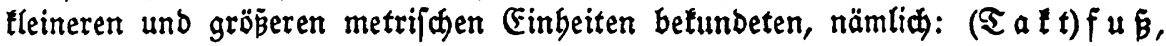
zujanmengejegt auz Grunbzeitwerten (chronoi protoi), $\mathfrak{P} \mathfrak{g} \mathfrak{r} \mathfrak{a}$ e (Rolon), zujanmengejeßt auz Saftfüßen, $\mathfrak{P} \in \mathfrak{r}$ i verjdiedenartigen Phrajengruppen, $\mathcal{S}$ tropge, zujammengejegt aus Perioden

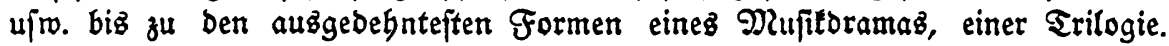
Uuf jeber Gtufe beruben bie Proportionen auf ben Prinjipien ber $3 \mathrm{we} i$.

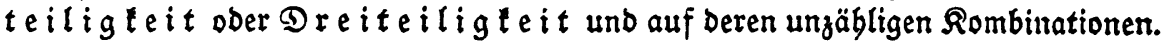

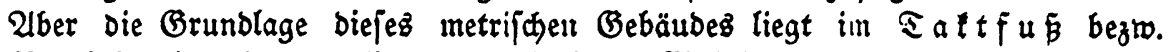
Oersfuß

Welchen Unteil bat ber metrijde Gü an ber modernen Mufiz? Die Beantwortung biejer Frage entideibet über bie Nugantwendung ber antifen Prinzipien auf bie Runft Der Begentwart. Die 2lnfitten ber Belegrten barüber geben auzeinander. Die einen bejagen biejelbe, die anberen verneinen fie. Die

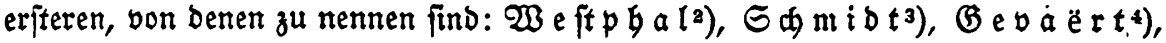

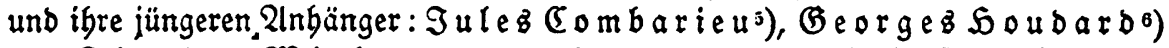

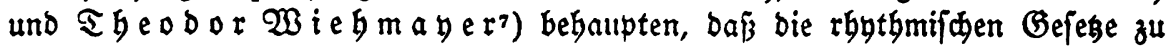
allen 3eiten biejelben bleiben. Dieje 2lnfint baben bie Gegner fdarf bejtritten, in ber Mleinung, daß bie moberne $\mathfrak{M u f i t , ~ i n s b e j o n d e r e ~ b i e ~ i n j t r u m e n t a l e , ~ v o n ~}$

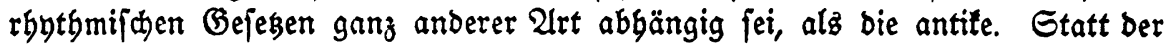

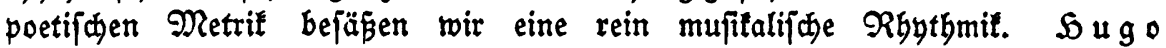
Ri e ma n'n'z bobe und Gerborragende 2lutorität Gat biejem Pringip bie Bevor. zugung jeitenz ber meiften beutigen Theoretifer verjळafft. Das Problem bes

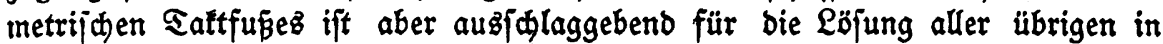
biefer Frage. Die Sauptiłtügpuntte ber negativen 2 rgumentation find folgenbe:

1) Bortrag, in franzöfil Mufit.Bejellimaft und im Original now nidit gebrudt.

2) Elemente Des mujitalijaen Rgythmus, 1872.

3) Die Formen ber griedif

1) Histoire de la musique de l'antiquité, $1875-81$.

5) Théorie du thythme, 1897.

i) Le rhythme du chant dit Grégorien, 1898.

i) Mupitnlifáe Rgytbmit und Metrit, 1917. 
1) ber Mlangel bez Grundzeitwertez (chronos protos) in ber modernen Mujit, und

2) Die 2luftattigleit Der mufifalijøen Motive.

Nod) ein brittez 2 rgument gegen bie 2 nnwenbung ber antifen Theorien auf

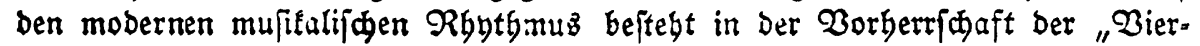
efigleit": "(carrure) in Der Formung Der Phrajen und Perioben und jteht fomit

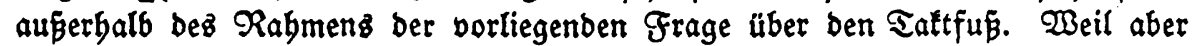
Daraus Ronjequenzen von hober Bebeutung für unjeren 3weá fíd zieben laffen, feben wir unz veranlaß̧t, auch diejez Urgument am Ende unjerer Erörterungen ou berübren.

\section{Der Grundzeitwert.}

1.

Waz ift der Grundjeitroert? Wir verjtegen Darunter die tleinjte Tondauer

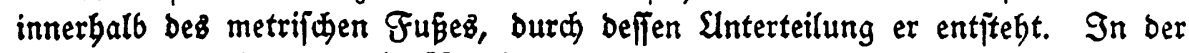
antifen Runft forberte bie Regel, baß er Der Dauer ber "lurzen" Gilfe ber Poefie entiprad, aljo ein Biertel beż gleid)en ober ein Drittel bez breiteiligen Taltfufez auzmad)te (= einer f-Note im $2 / \%=$ ober $3 / 8=$ Talt). Ez war nid)t geftattet, bie Dauer ber Töne Durd noch weitere Slnterteilung zu verlleinern. Die moberne Mufil bat fī aber, bebauptet man, von biejer Finzrängung

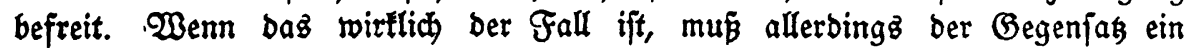
bebeutender fein.

Sndeffen war jene Regel in ber antifen Mufif nidjt unbeugfam. (Es bejtanden

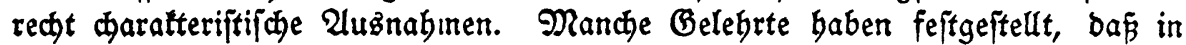

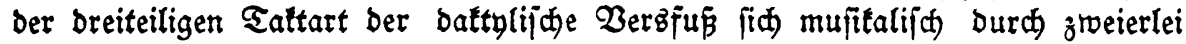
Rgytbinen auborüđtte, bie Söne von fürzerer Dauer entbielten, alz bie obige

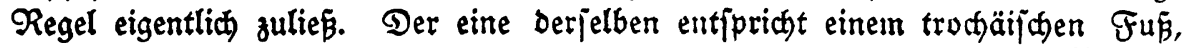
Deffen leidter furzer Taftteil in zrwei gleidjlunge Töne geteilt iłt:j E; Der andere läß̈t fíd vom Tribradyz ableiten, beffen zwei erften Tönen ibre gemeinjame

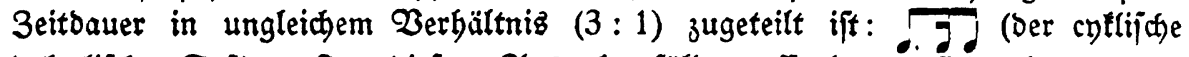

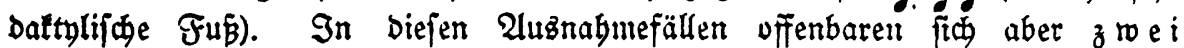

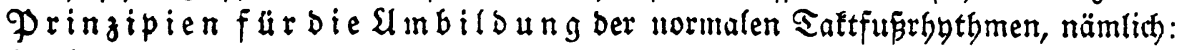
Roloratur und $\mathfrak{x y}$ thmifde alteration.

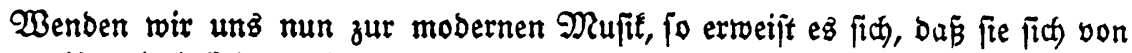
ber antilen lebiglid burd a a gebehnteren und fonfequenteren

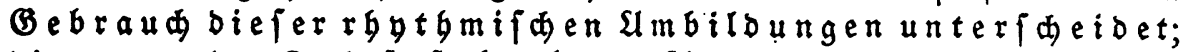

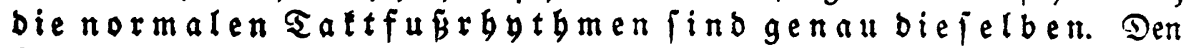
Berweiz Dafür möđten wir im folgenden liefern.

II.

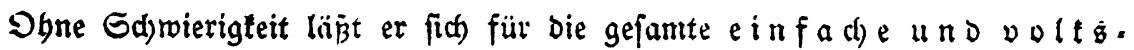

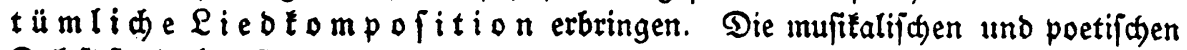

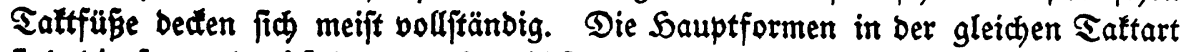

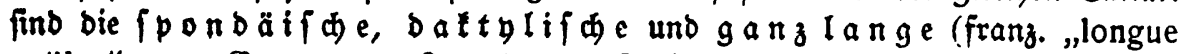

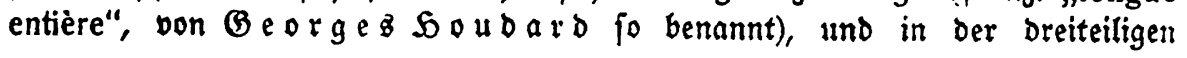




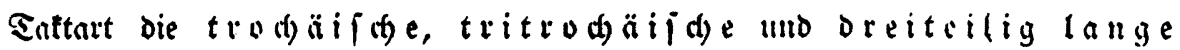
(fratj. „longue ternaire") oder je nad) ber Gatreibart:

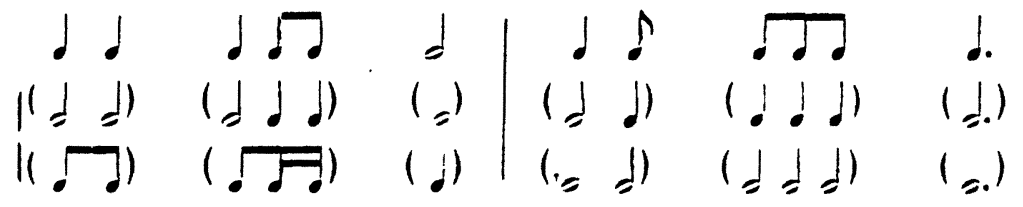

Dur flürthtigjte 3 lidf auf jede beliebige Riebjammlung wird baz bejtätigen. Neben

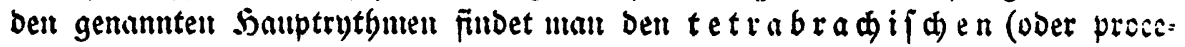

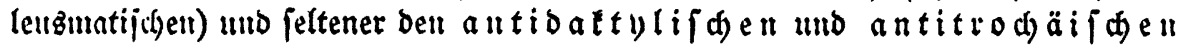

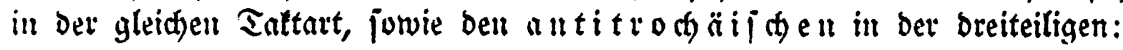

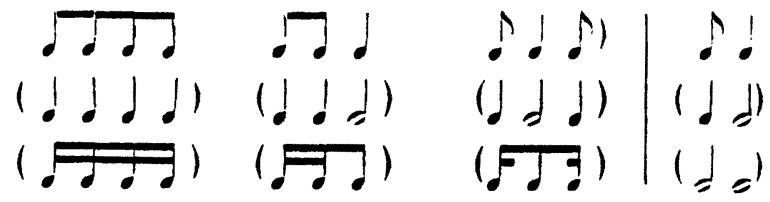

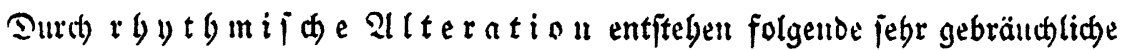
Sattfübe: ber alteriert fponbäif

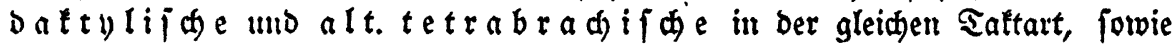

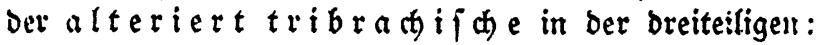

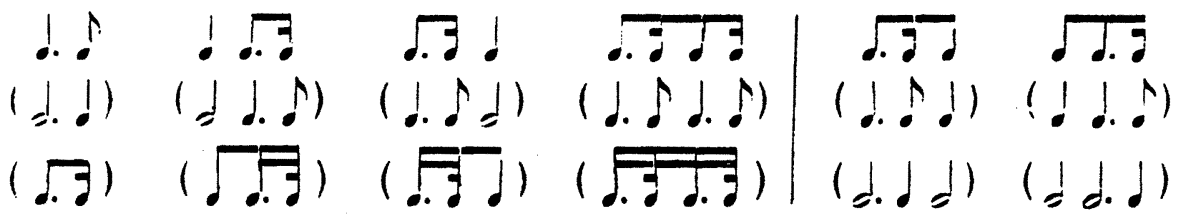

Die $\mathfrak{b}$ e ge $n \mathfrak{a} \mathfrak{l}$ e $\mathfrak{x}$ ation $(1: 3)$ ift überauz felten in einfaden Melobien

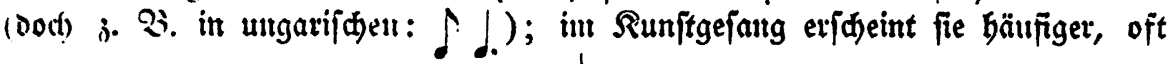
aber alz "Burfallag" bezeidanet: $\xi=$ ).

Dii Sioloratur=Rbytbmen, bie eirten wejentlichen Beftanbteil ber entrvidetelteren Monobie auzınadien, find unzäglig. Sie beziebent fid jebod in ber

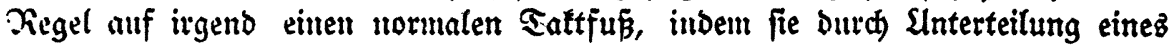

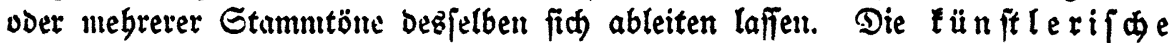

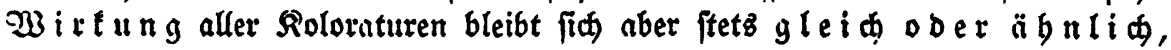

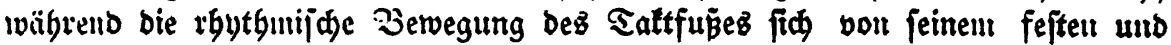

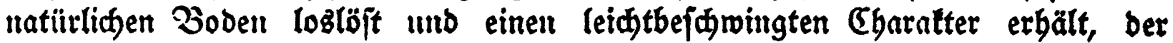

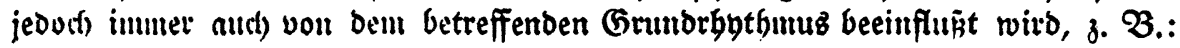

\section{Dakt. . F! toloriert! u[w.}

III.

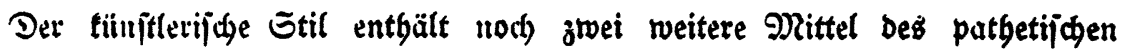

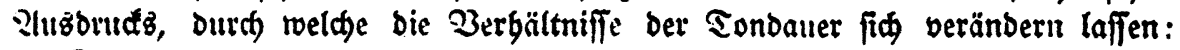
Die $3 i$ u 


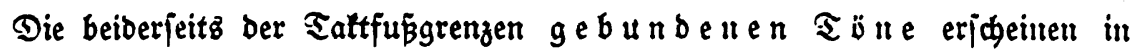
allen mögliden Rombinationen; aber fie werben ftetz alz aupergemöbnlidise

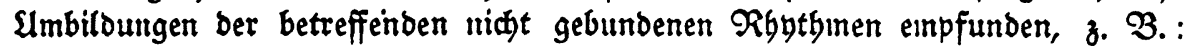

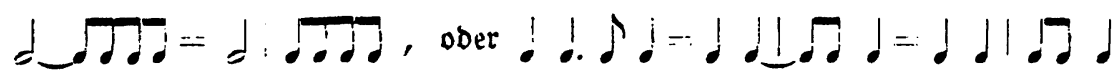

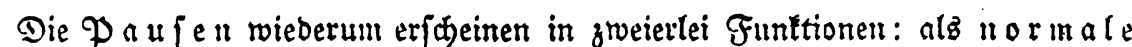
voer pathetifde. Die erfteren finben fíl an ber Grenze ber Phrajen ober Phrajengruppen. Ullzbann berbollfändigen fie bie Tonbauer ber $\mathfrak{t}$ a $t$ ale $\mathfrak{f}$.

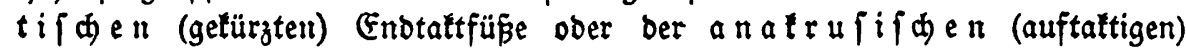
Unfanggitnltfü̈ße, z. $\mathfrak{B}$.

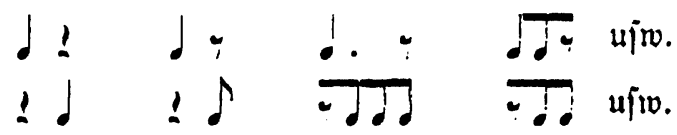

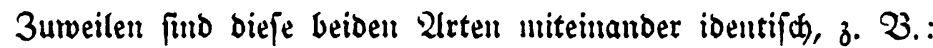

$$
\text { ! y } j \div \text {. }
$$

Dieje Paufen gehören zum regelrechten Gejtanbteil bez metrifa)en (Sefügez ebenjowobl in ber antifen alz in ber mobernen Mufif. Sie pathetifd)en Paujent erfdeinen nur auberbalb ber einfacberen Mufit und tragen aud) einen ganj anderen Charafter. Mitten in ber Phraje verurfachen fie eime Staccato=

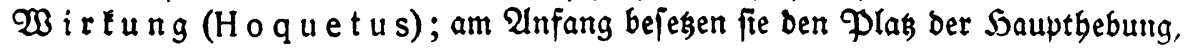

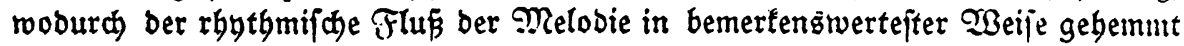
iviro (prototalettifhe $\mathfrak{P h r a f e ) . ~ S t e t z ~ a b e r ~ l a f f e n ~ f i e ~ j i d s ~ a u f ~ e n t i p r e d i e n d e ~}$

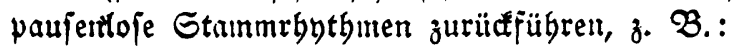

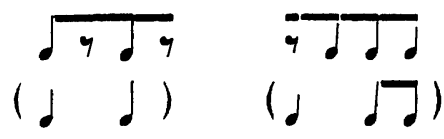

IV.

Sn ber $\mathfrak{I}$ utrumentalmuji furb bie melodiefülrenden Etimmen von Den begleitenden ju unterjdeiden. Die erjteren folgen ben

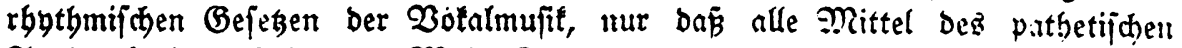

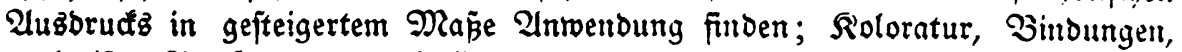
patbetijde Paujen treten reid)licher auf. Die meiften Suftrumentalthemen lajient jidh leidht auf Die metrif jelben ourd) tolorierende Unterteilung und Bittoung ober Slnterbred)ung Der nor= malen Sonlängen mittelijt Paujen. Dem entipridt audh bie jelveilige äjtgetijde Wirtung. Die Etamniformen der Saltfü̈̈e jumb gezäglt und baben jebe igren

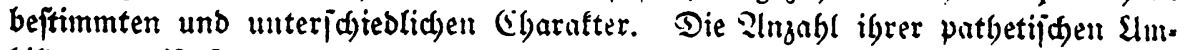
bildungen ift jo gut wie unendich; bod) verleigen jie bem mujitulijchen (Gbarafter nur cine leidte Eonberfärbung gemä̈ ibrer allgemeinen Sintegorie von Rolorntur, Binbung ober \$aufemunterbrect)ung. 
In ben begleitenden Snltrumentalftimmen Gaben die Slmbitbungäformen Geutzutage fajt bie Borberridaft. Die Runjt bez Snjirumentierens, bie in ber Mufit ber Gegenwart wohl ben Sö̈hepunlt igrer Bollendung erreidt Gat, f内öpft

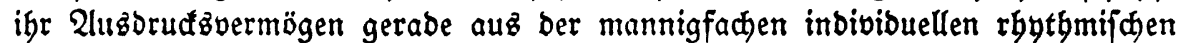
SImbilbungżart, bie jebem Snjtrument ober jeber Injtrumentengruppe bejonderz eignet. 2llle bieje ragthmijden Bildungen, biż zu ben verwideltften, ruben

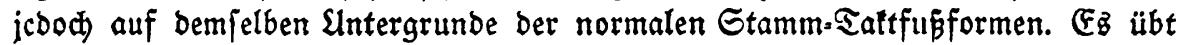

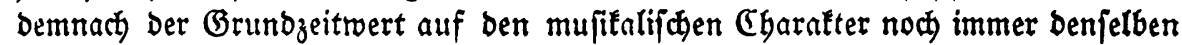

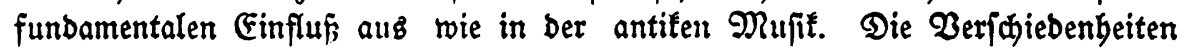
liegen bló̉ auf ber Oberfläche und verbanfen ibr Dajein megr einer Ebo= lution, benn einer Rebolution.

\section{Der $\mathfrak{A} \mathfrak{u} \mathfrak{f t a t t}$.}

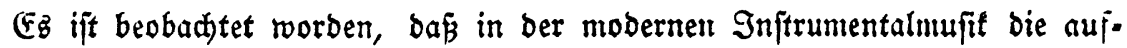
taftigen Gebilbe nidjt nur in ben Phrajen, fonbern audi in ben fleiniten Motiben unb Teilmotiven (incises) vorberriden ( $R$ i e $\mathfrak{m} \mathfrak{a} \mathfrak{n} \mathfrak{n}, \mathfrak{L} \mathfrak{u}[\mathfrak{j} \mathfrak{y}$ u. a.). Daraus

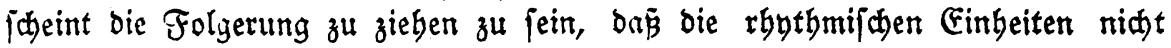

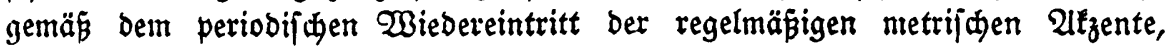
fondern je nadh Dent verjaiedenen Rombinationen von Genfung und Sebung zu meffen fint. aber ba bie Möglidfeeiten ber rhytbmijळen 3 ujammenjegungen bierburdh fich faft zahl= unb grenzenloz vermegren, verlieren fid bie Prinzipien

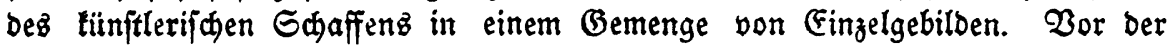
unerjळöpfliden Snjpiration ber Sonjeger bätte Der SGeoretifer fomit nur zu

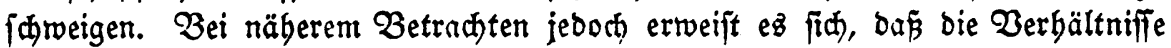
in ber Poefie Durchauz äbnlich liegen, indem bie Worte unb Wortgruppen

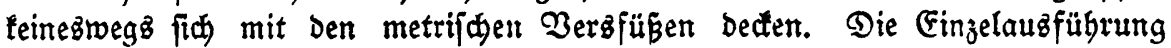

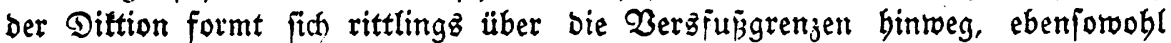
wie bie mufitalijanen Motive über bie Sattifriche. Deffenungeadytet ift baz

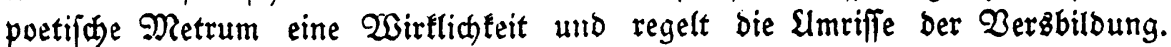

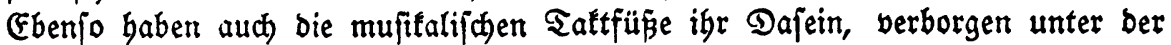

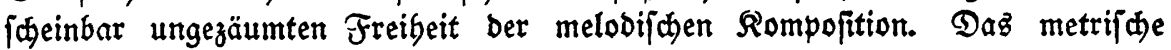
Prinzip fommt pogar noch anfdaulider in ber Tontunft zutage, alż in Der

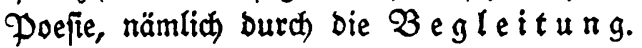

Die regelmäß̈igen Sobungen, bie gemäß ben Taktfüß̈en und Pgrajen bie

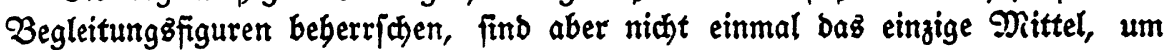
bie metrijळe Grundage ber Siumpojition hervortreten zu laffen. Der Wechjel

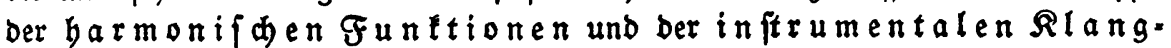

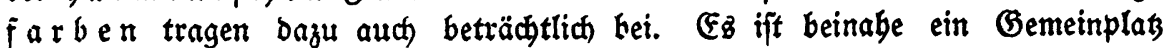
feftzuiftllen, bas bie grunblegenden Soarmoniejdritte fidh au bie metrijđen Sebungen balten; aud baben mandie Ordefterinjtrumente öfterz einzig baż metrifade Gefüge bez Tonjakez zur Beltung zu bringen.

Gomit ift, trog aller (Finnänbe, bie antife Qegre über Taftfüße und Phrafen Geranzuziehen, um bie rhytgmijhen Proportionen ber augigebebnteften mobernen Injtrumentalformen theoretifich zu ermitteln und auseinanderzufezen. Bon biefer 
foliben Grunblage auz erlangt man einen umfaffenden äberblif über igre ver: wideltiten Erideinungen, obne Damit ber Freibeit ibrer Entwia̛lung einen 3rang anzutun.

\section{Die Bieredigteit.}

I.

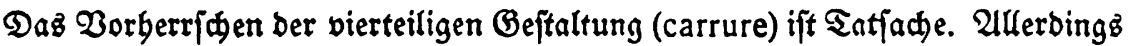

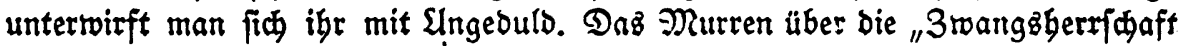
Dez Taltez" richtet fich eigentlich nur gegen bie Bieredigleit ber Form. Woburd) wirb fie aber wejentlid bebingt? Rur了 und bündig geantrovtet, gebören zur "Wiereçigfeit" folgende Merfmale:

1) $\mathfrak{g} \mathfrak{l}$ e

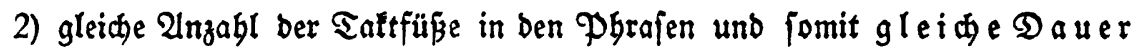
ber $\mathfrak{P} \mathfrak{b} \mathfrak{r}$ ajen;

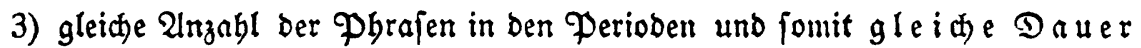
der Derioden.

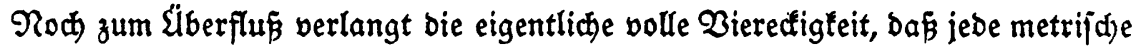

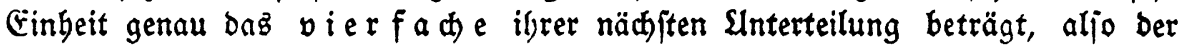

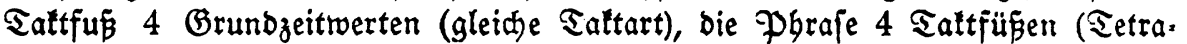
podie), die Periode 4 Phrajen (gepaarte Normalperiobe) entfpriat. Die vier

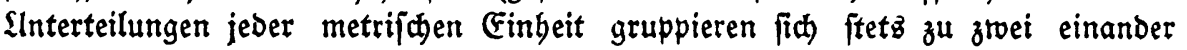

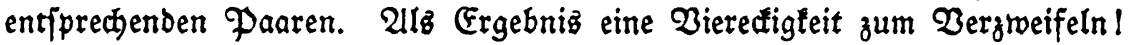

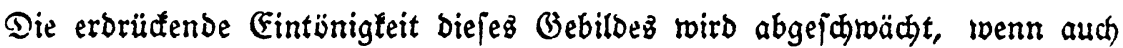

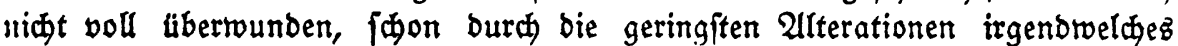
feiner Brundelemente. Die meiften gegentwärtig gebräudblichen Rompofitionzlebren begnügen fid mit ber 2lngabe veridiebener $\mathfrak{A}$ rten von Ulterationen, bie ben

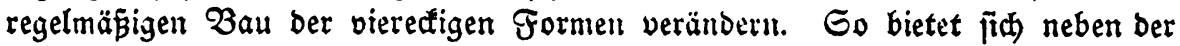
gleidhen Taltart bie oreiteilige; bie vierbebige Beftalt ber Phraje verfürzt

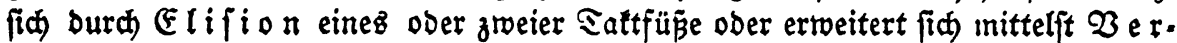
boppelung ber Grundzeitwerte einez ober mebrerer Taltfübe; bie

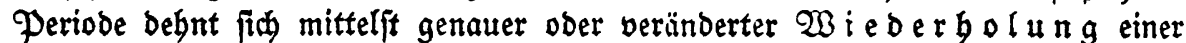
Whraje oder Phrajengruppe ober aud) burd) gelegentlidez (F inf ügen einleitender, vermittelnder und abjaließ̈ender $\mathfrak{P}$ grajen (proodikon, anesodikon, epodikon).

Das allez ift wohl geeignet, bie Bierectigteit ju verbefen, aber nidit aus. reidend, um fie zu fprengen. Slnd bod bäumt fidj baz mufitalijche Cimpfinden auf gegen jenes 3ranggjod, um über alle metrijळen Mittel frei verfügen zu tönmen, burd welche das innere Leben ber Runjt in Erfd)einung treten wial.

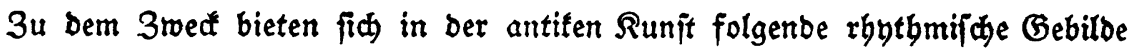

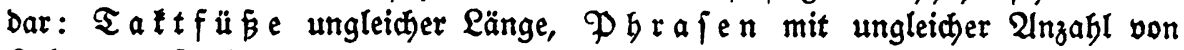

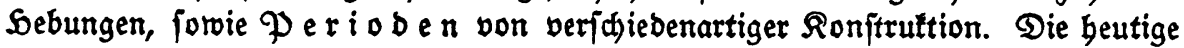
Mujit bejigt bieje Runjtmittel ebenjorwobl; nur werben fie in ber Notenjarift nidt beranfdaulid)t und bleiben in ben Qebrbüdern unerwäbnt. 
II.

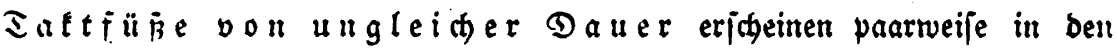
jouij(ben unb pä $3 / 8+2 / 8$ obcr $=/ 8+3 / 8$, bejw. $3 / 4+2 / 4$ ober $2 / 4+3 / 4$ ). Diejelben fünnen als

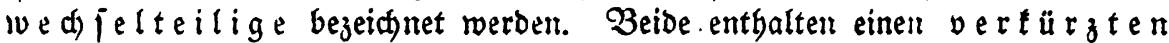

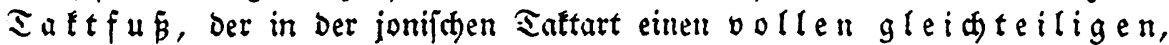

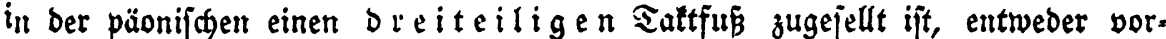
bergebeno oder nachjolgeno. Der verfürjte Tattfü ijt aber einem $\mathfrak{h}$ a gleich teifige n bur(baus ägnlich und vertrïgt bie entjprewenden alterationen

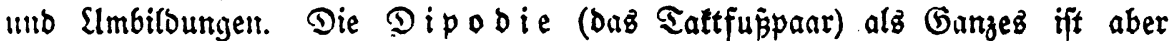

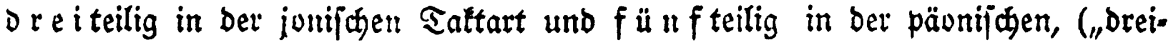
weedjelteilig", "fünfroedjelteilig"). In ber Notenjarift ift man genötigt, bieje Saltarten ftetz nac() Dipodien zu bezeichnen $(3 / 4$ und $5 / 8$, bezw. $3 / 4)$. Es entpfieblt

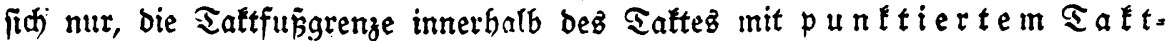

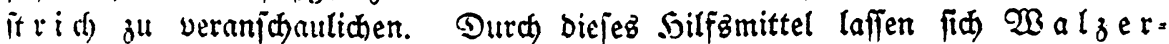

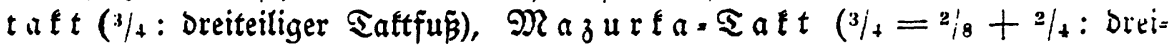
ivechjelteilige (Dipodie) und $\mathfrak{P}$ o l o n a i f e n talt $(3 / 4=2 / 8+2 / 8+2 / 8$ : Tripodie

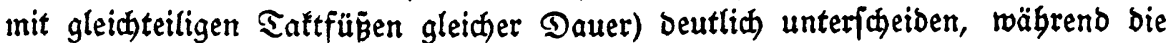
übliche Notierung fie miteinanber vermengt.

III.

Die Somptrirfung ber "vierecfigen" Formung wurjelt jebo(t) in ber $v$ i e $r=$

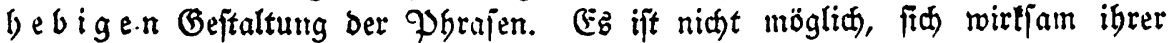
(3etvalt zu entwinben, auß̧er burch Vlnerfennen ber übrigen Saftfü̈gruppierungell:

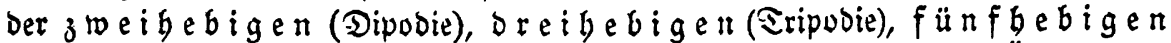
(Petrtapobie) und f ech ż

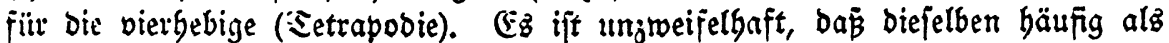
ivejentlidje Bejtunbteile ber mujfalif̧en Ronzeption ber Neifterwerle auftreten.

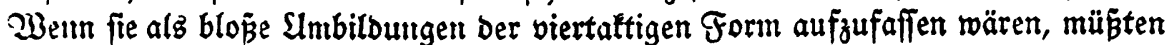
fie fich Durch Setrapodien erfegen laffen, obne die Brunoproportionen ber Rompo.

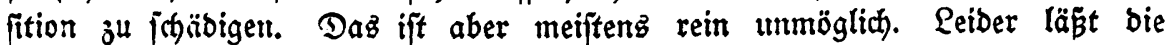

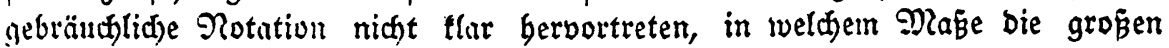
Meifter, trus ber unjulänglichen SGeorie, von nidjtbierbebigen Phrajen Gebraud

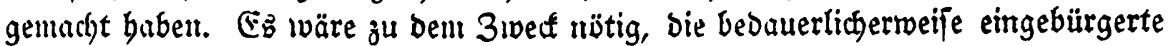

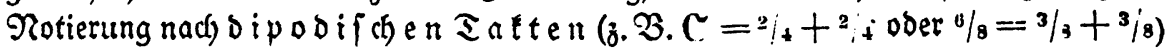
anfigeben, Deren Sinwendung bie Whantafie Der Romponiften an bie gleicteiligen

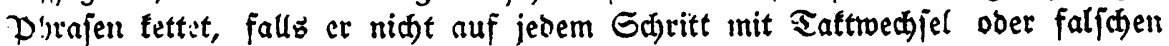
Saltitrichen fits quälen ober mit mebr ober weniger zweibeutigen Fermaten aubtommen will. Slbyelolfen wirb biefem S̈beljtand nur burch allgemeine $2 / n=$ nabme monoposifder Iafte (Iattfü) - auggenommen bie rochjel teiligen, benen ber punftierte Gtrich abzubelfen bat - powie mittelft befonderer

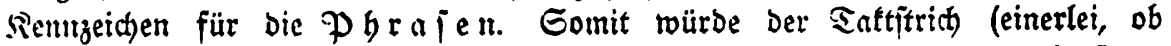

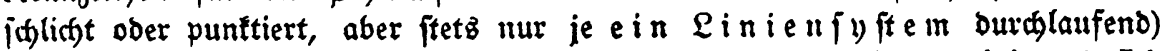

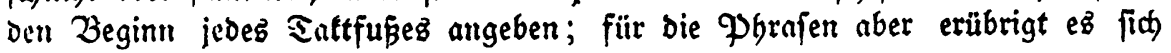




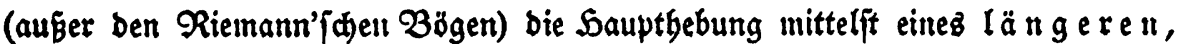
zwei ober mebrere Gyfteme burdmeffenben Taltfitridez zu bezeidjent ober aud).

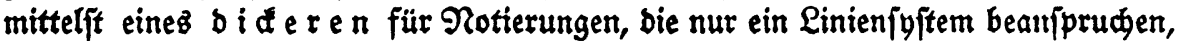
ober eines $g$ ef $\mathfrak{a} \mathfrak{r} \mathfrak{b} t$ e $\mathfrak{n}$ für gefdriebene Ordefterpartituren, wo alle Saltftridje ber Bequemlidteit balber über bie ganze Geite zu zieben find. Fine Hleine

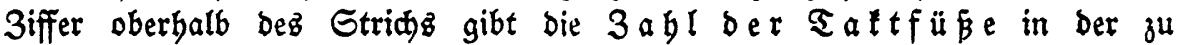
beginnenden Phraje ${ }^{1}$ ) an, z. $\mathfrak{B}$.:

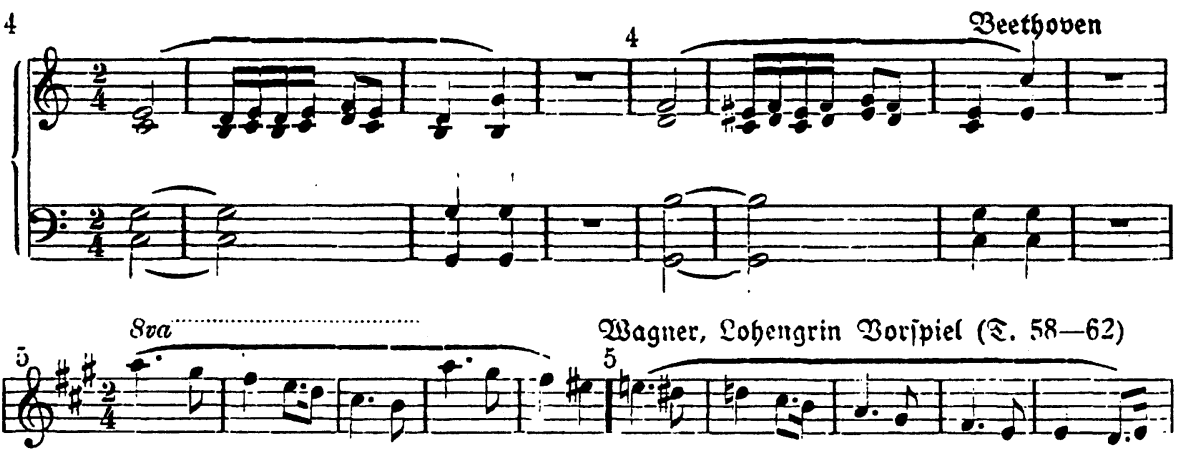

IV.

Die Perioben Der fluffifden Meifterwerle entfernen fich weit won Der Biereçigleit. Slm ben angebenben Runitjüngern zum vollen Berf́tünoniz ber fompofitorijaen Proportionen $z^{u}$ verbelfen, beren unmittelbare Künjtlerijche Wirtung fie unberoujt empfinden, ift es nötig, bie antife Periobenlegre beranzuzieben. Bei eingebender 2lnalyje moberner Tonwerfe wirb man jomit beobaditen tönnen, wie bie pali no bif de ("gepaarte", ober in ifrer allerein. fachjten Form rein "pierectige") Etruftur ber $\mathfrak{l}$ y $x$ i $\{$ क e $\mathfrak{n}$ (Fnipfindung entipridt,

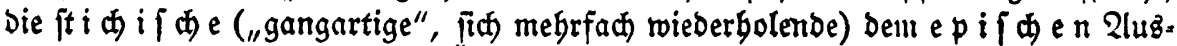

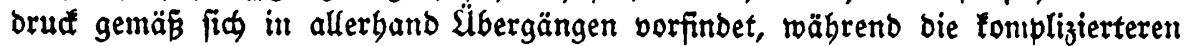

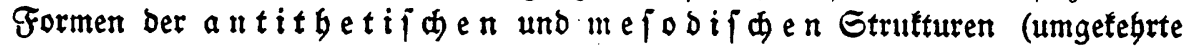
Reibenfolge ber äquibalenten Phrajen ober Phrajengruppen, z. $\mathfrak{b}$. a b b a, ober Erifheinen einer felbjtändigen Phrafe ober Gruppe zwifden zrei einander entipredjenden, z. 3 . a b, c, a b) bem brama tij licjiten fich erroeijen. Den $\mathfrak{B}$ eleg für bieje begauptung wirb man $\mathfrak{u}$. $\mathfrak{a}$. in

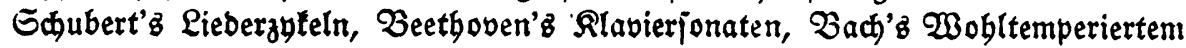
Rlavier unb Wagner'z Lohengrin in überrajめender Weife vorfinben. Der Finzelanalyje biefer Werfe bat Interzeidnneter bie Geminumng feiner S̈berzengung zu verbanten.

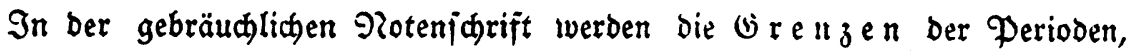
jorwie ber Etrophen und anderer ausgedebnterer rghthmif her (Einbeiten, nur

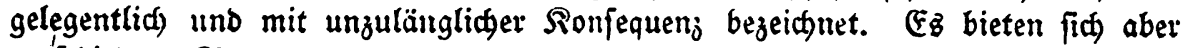
verfdiebene 2Irten bon Doppel- und breifaden Etridjen bequem Dazu Dar. Go tönte ber einface Doppelftrid in jeber Periobe ben erften

1) G. Deż Berfalfers: „Rejorm Der Saltbezeidyuny", Ill. Rongreß Der IMG, 1909. 
Pgrajenftrid erfegen; für bie Phrajengruppen innerbalb ber Periobe gentigen Die Riemann'¡đen "Lejezeid)en" ober bejonbere ben Pgrafenziffern beigefügte 3eiden, z. B. V. Beim Cintritt einer neuen Gtrophe wäre einer ber Gtride

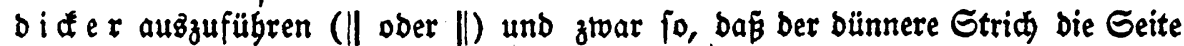
Der Sauptftrophe coer beren Repetition bezeidnet, ber biafere biejenige ber

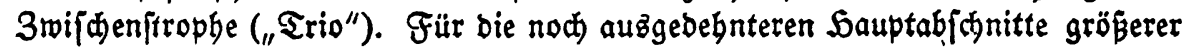
Tonwerle bieten fid neben bem boppelten bifien Gtrid (II) aud veridieden tombinierte breifache Gtridje (z. $\mathfrak{B}$. |l| oder ||I).

Die vorgelegten 2luz̧führungen bezweđen nidt leere theoretifळe Epelulationen.

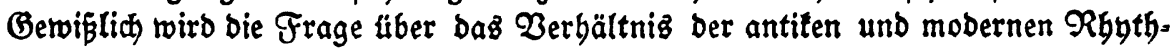
mit, in pofitiver Ridtung entichieben, eine frudtbare Anregung fowohl für baż Berftänoniz ber bereitż vorliegenden Tontwerle, alz auch für bie zutünftige

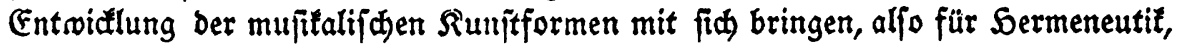
Bortrag und Rompofition wertboll pein. 